\title{
Recent Advances in the Development of Animal and Cell Culture Models for Prostate Cancer Research
}

\section{A Minireview}

\author{
Maarten C. Bosland, Leland W.K. Chung, N.M. Greenberg, Shuk-mei Ho, John T. Isaacs, \\ Kate Lane, Donna M. Peehl, Timothy C. Thompson, Gert J. van Steenbrugge, \\ Wytske M. van Weerden
}

From the Departments of Environmental Medicine and Urology, New York University Medical Center, New York, NY, USA; Molecular Urology and Therapeutics Program, Department of Urology, University of Virginia Health Sciences Center, Charlottesville, VA, USA; Department of Cell Biology, Baylor College of Medicine, Houston, TX, USA and Scott Department of Urology, Baylor College of Medicine, Houston, TX, USA; Department of Biology, Tufts University, Medford, MA, USA; The Johns Hopkins Oncology Center, Baltimore, MD, USA; Department of Urology, Stanford University School of Medicine, Stanford, CA, USA; Department of Urology, Division of Urologic Oncology, Erasmus University, Rotterdam, The Netherlands

A ccording to U.S. cancer statistics, prostate cancer has been the most commonly diagnosed cancer and the second leading cause of cancer deaths in American men. ${ }^{1}$ To improve the diagnosis and the clinical management of this disease and to prevent its future occurrence, a number of strategies need to be implemented. One strategy is to develop relevant animal models and tissue culture systems that closely mimic clinical conditions of human prostate cancer. This could help us develop new diagnostic tools to distinguish the indolent from the virulent forms of the disease and to design novel therapeutic approaches to target this disease. Such efforts could improve the prevention, diagnosis, prognosis, and treatment of prostate cancer.

This minireview summarizes the relevant animal models and tissue culture systems with comments from leading authorities who made the original contributions to the prostate cancer model systems described. This review is intended to: (1) summarize pertinent published literature on the nature of the prostate cancer model described; (2) discuss the strengths and the limitations of the model systems in answering important biological questions facing prostate cancer research; and (3) provide source information about where these prostate cancer models can be obtained. The contributing authors have a number of goals. First, we hope that this article will provide relevant and accurate background information to an increasing number of new investigators whose research has begun to focus sharply on prostate cancer biology and therapy. Second, we hope to attract the interest of new investigators to devote their talents to this fertile area of cancer research. Finally, we believe that using experimental model-based prostate cancer research will increase rapidly our understanding of the fundamental aspects of prostate cancer biology, including its development, progression, differentiation, and senescence. The basic knowledge gained from these fundamental studies can be translated to the clinic to improve our ability to prevent, diagnose, prognose, and treat patients with prostate cancer.

\section{Reference}

Address correspondence to: Dr. Leland W.K. Chung, Department of Urology, University of Virginia Health Sciences Center, Box 422, Charlottesville, VA 22908 .
1. Parker SL, Tong T, Bolden S, Wingo PA. Cancer statistics. CA Cancer J Clin 1996;46:5-27. 